\title{
A novel elevated plus-maze procedure to avoid the one-trial tolerance problem
}

\author{
Peggy Schneider', Ying-Jui Ho ${ }^{2}$, Rainer Spanagel ${ }^{1}$ and Cornelius Rainer Pawlak ${ }^{1,3,4 *}$ \\ Institute of Psychopharmacology, Central Institute of Mental Health, Mannheim, Germany \\ 2 School of Psychology, Chung Shan Medical University, Taichung City, Taiwan, ROC \\ ${ }^{3}$ Department of Cognitive and Clinical Neuroscience, Central Institute of Mental Health, Mannheim, Germany \\ ${ }^{4}$ Department of Addictive Behavior and Addictive Medicine, Central Institute of Mental Health, Mannheim, Germany
}

\section{Edited by:}

Riccardo Brambilla, San Raffaele Scientific Institute and University, Italy

\section{Reviewed by:}

Kerrie L. Thomas, Cardiff University, UK Thomas Burne, Queensland Brain Institute, Australia

*Correspondence:

Cornelius Rainer Pawlak, Central Institute for Mental Health, J 5, 68159

Mannheim, Germany.

e-mail: cornelius.pawlak@zi-mannheim. de
The elevated plus-maze (EPM) test is one of the most commonly used behavioral assays to evaluate anxiety-related behavior in rodents. It is an economic test (5 min duration) without prior conditioning of the animals. The critical measure for anxiety is the time spent in the open arms of the maze. A confounding problem of the EPM is the so called one-trial tolerance (OTT), characterized by a marked decrease of open arm exploration in spite of treatment with anxiolytic acting benzodiazepines upon re-exposure to the EPM. This consistent finding is often raised as an evidence for the inappropriateness to re-test rodents in the EPM. However, a reliable re-test paradigm would broaden the usability and effectiveness of this test. Therefore, we tested how an extension of the inter-trial interval to 28 days (instead of the usual $24 \mathrm{~h}$ ), and an additional change of the testing room would affect the open arm time and other behaviors on the EPM. In two experiments, drug-naive Wistar rats were exposed to the EPM on trial 1, and treated intraperitoneally with either vehicle or midazolam $(0.25 \mathrm{mg} / \mathrm{kg}) 30 \mathrm{~min}$ before trial 2 . Then, trial 2 (28 days after trial 1) was carried out in either the same testing room (Experiment 1) or in another unfamiliar room (Experiment 2). Twenty-eight days after trial 1 the open arm time of the rats in the vehicle treated control rats of both experimental groups was comparable to that of the first trial, independent of the testing room. Most importantly, we found that the treatment with the benzodiazepine midazolam had a significantly anxiolytic-like (i.e., increase of open arm time) effect in trial 2 only when conducted in the previously unfamiliar testing room (Experiment 2). We suggest that in order to reliably re-test the EPM and to prevent confounding effects due to the OTT, an inter-trial interval of 28 days and a change in testing rooms reinstates anxiolytic-like actions of benzodiazepines.

Keywords: re-test EPM, one-trial tolerance, midazolam, motivation, memory

\section{INTRODUCTION}

The elevated plus-maze (EPM) is one of the most commonly used models in behavioral neuroscience to evaluate rodent anxietyrelated behaviors. The EPM is validated for rats (Pellow et al., 1985) and mice (Lister, 1987; for review see Carobrez and Bertoglio, 2005; Walf and Frye, 2007; Pawlak et al., 2008).

Even though the EPM paradigm has been extensively used there are still aspects which remain inconclusive, most prominently the phenomenon of "one-trial tolerance" (OTT), first described by File et al. (1990). The OTT is characterized by a marked reduction in the anxiolytic-like effect of benzodiazepines (BDZ) during a reexposure to the apparatus after only one previous EPM experience (File and Zangrossi Jr., 1993; Gonzalez and File, 1997; Frussa-Filho et al., 1999; Pereira et al., 1999). An increase in anxiety-related behavior has also been found in other animal models for anxiety, such as the light/dark emergence test (Holmes et al., 2001), and the mouse four-plate test (Hascoet et al., 1997). Further investigation showed this effect to be independent of treatment with BDZs on the first trial, and it can be found with inter-trial intervals from $24 \mathrm{~h}$ up to 14 days (File et al., 1990; Rodgers et al., 1992; Rodgers and Shepherd, 1993). Environmental changes, e.g., lighting condition, position of the maze, the material of the apparatus, white noise level, or change in laboratories, did not affect the OTT in a re-test situation with $24 \mathrm{~h}$ interval time (Rodgers et al., 1997; Calzavara et al., 2005). Moreover, variation of the time span of the first trial results in often contradictory data across the literature (File et al., 1993; Holmes and Rodgers, 1999; Dal-Col et al., 2003; Calzavara et al., 2005).

It has been suggested that the prior experience of the EPM causes a shift of the emotional state of the animal and the acquisition of a phobic state instead of an unconditioned anxiety response (File et al., 1993; Cruz-Morales et al., 2002). As BDZs are anxiolytic but not effective in phobic-like states this might explain the lack of reactivity in the second EPM trial (Lister, 1987; File, 1993; File and Zangrossi Jr., 1993). This emotional shift has been a main focus of the research concerning the OTT and the re-test reliability of the EPM test as the accumulated literature indicates that a second trial appears to measure fear rather than anxiety (Pawlak et al., in press). These changes in the behavioral profile have also been assessed statistically using factor analysis to assign measurements taken to a number of factors underlying concepts such as anxiety, risk assessment, or fear. The analysis of trial and re-trial revealed 
that the measurements of the anxiety level change in valence (i.e., from anxiety to fear) across the two sessions, further indicating an emotional shift (File, 1993; File and Zangrossi Jr., 1993; Holmes and Rodgers, 1998; Albrechet-Souza et al., 2008). The analysis of so called "novel" ethological parameters, i.e., head dips, rearing, and risk assessment, adds an additional dimension to the behavioral profile shown on both exposures to the plus-maze and allows for further evidence for the shift in the affective state of the animal (File and Zangrossi Jr., 1993; Rodgers et al., 1996).

Another aspect is the knowledge which is gained during the first trial. In the course of exploring the entire maze the animal loses the acute approach/avoidance conflict it has initially shown (Rodgers and Shepherd, 1993). However, when introducing an aversive stimuli in the closed arms on trial 1, Pereira et al. (1999) could retain a motivational conflict situation, which resulted in an anxiolytic-like effect (i.e., increased open arm time) when treated with BDZs. This evidence points to a spatial as well as an emotional learning process during the first trial that is responsible for the changes in behavior from trial 1 to trial 2 in the EPM (Rodgers et al., 1996; Lamprea et al., 2000; Rosa et al., 2000).

The OTT is often taken as one critical evidence that the EPM measures two different concepts of aversively motivated behaviors upon re-testing and would therefore reflect state- rather than trait-anxiety (Andreatini and Bacellar, 2000). On the other hand testing EPM behavior is the basis of the established breeding lines of high and low anxiety-related behavior Wistar rat lines, which show a distinct phenotype profile and point to trait rather than state anxiety measured by the EPM (Landgraf and Wigger, 2002). Further evidence for measuring trait behavior on the EPM is the positive correlation of open arm time measurements in unselected Wistar rats between two sessions in the EPM with inter-trial times ranging from $24 \mathrm{~h}$ up to 120 days (Schwarting and Pawlak, 2004). This stability in behavior over time, especially because the open arm time recovers after 120 days, supports the categorization into high open arm (HOA) and low open arm (LOA) rats (for review see Pawlak et al., 2008).

Although the EPM is an economic test paradigm, a simple reexposure after $24 \mathrm{~h}$ (and even up to 14 days later) presumably measures different qualities of aversively motivated behavior. While trial 1 is often suggested to reflect a form of anxiety, trial 2 appears to measure fear-like avoidance behavior in rodents. A re-test paradigm which would permit longitudinal studies has yet to be established. Such a reliable, valid, and easy to use re-test paradigm would substantially widen the advantages of the EPM test by allowing a re-exposure of rats to the EPM without eliciting the OTT phenomenon. Thus, it was the aim of our experiments to obtain results comparable to the behavior of the first trial. We hypothesized that the combination of a prolonged inter-trial time and a change of the testing environment would abolish the OTT effect of midazolam and thus retain the measurements of baseline anxiety-like levels.

\section{MATERIALS AND METHODS ANIMALS}

Male out bred Wistar Unilever rats weighing 220-249 g each were obtained from Harlan (Eystrup, Netherlands) and allowed to habituate for 2 weeks after arrival at the lab. The animals were housed in groups of four under a light/dark $12 \mathrm{~h}$ cycle (lights on from 0800 to $2000 \mathrm{~h}$ ) with free access to food and water. All experiments were conducted in accordance with the ethical guidelines for the care and use of laboratory animals, and were approved by the local animal care committee (AZ: 35-9185.81/G-107/07; Regierungspraesidium Karlsruhe, Germany).

\section{APPARATUS}

The EPM was made of dark gray PVC consisting of two opposite open arms $(50 \mathrm{~cm} \times 12 \mathrm{~cm})$ and two opposite closed arms surrounded by $50 \mathrm{~cm}$ high walls of the same dimensions. The middle section that allows the animal to transit from arm to arm consisted of a square with dimensions of $12 \times 12 \mathrm{~cm}$. The maze was elevated $50 \mathrm{~cm}$ above ground and the open arms were equipped with $0.5 \times 0.5 \mathrm{~cm}$ ledges to ensure that no animals would fall off the maze. The apparatus could be moved between rooms and it was made sure that placement and lighting conditions were identical for each trial. The trials were video recorded and computer analyzed with the ethological software viewer ${ }^{2}$ (Biobserve $\mathrm{GmbH}$, Bonn, Germany) to measure time spent in and visits to the arms of the EPM. All other ethological analyses were performed by an experienced observer. The PC equipment was located outside of the experimental rooms. All experiments were carried out during the middle of the light phase between 1100 and $1600 \mathrm{~h}$.

\section{DRUG}

Midazolam (ratiopharm, Ulm, Germany) was obtained as solution suitable for injection and freshly diluted as needed with $0.9 \%$ saline. All solutions were administered intraperitoneally (i.p.) $30 \mathrm{~min}$ prior to the EPM trial in a volume of $1 \mathrm{ml} / \mathrm{kg}$.

\section{PROCEDURE}

All trials were carried out under low light conditions (30 lux white light in the center of the maze) in either of two unfamiliar experimental rooms. Room 1 (R1) and Room 2 (R2), which mainly differed in size, with R2 being the larger one, but with the same basic make-up (white walls, identical light equipment, and comparable room equipment). Both experimental rooms were similarly distanced from the animal housing room. Each rat was gently placed in the center of the maze always facing the same open arm. All trials lasted $5 \mathrm{~min}$ each allowing the rat to freely explore the EPM. Between each animal the maze was thoroughly cleaned with a $30 \%$ ethanol solution and dried afterward. All experiments were carried out by the same experimenter which has been shown to be critical in reducing data variability (Chesler et al., 2002; Wahlsten et al., 2003).

\section{PILOT EXPERIMENT}

Two groups of 18 drug-naive rats each were tested on the EPM (trial 1) in the room later referred to as R2 (see Experiment 2: Switched room). After the first trial the animals were returned to the housing room and remained there for 14 days. Following this time period each group of animals was again tested on the EPM for a second trial: one group was tested again in the same room (R2), and the other group in a new testing room later referred to as R1 (see Experiment 1: Same room). The animals were tested drug free on both trials. 


\section{DOSE-RESPONSE EXPERIMENT}

The initial dose-response experiment was carried out with a separate group of 32 Wistar rats. The drug-naive animals were randomly assigned into the four treatment groups $(n=8)$ receiving either $0.25,0.5,1.0 \mathrm{mg} / \mathrm{kg}$, or saline. The trials were carried out in the room which is referred to as R1 in the following experiments. Following this $0.25 \mathrm{mg} / \mathrm{kg}$ was used in the subsequent EPM experiments as the most effective dose.

\section{EXPERIMENT 1: SAME ROOM}

A group of 20 drug-naive rats were tested on the EPM in room 1 (R1). After this first trial the animals were brought back into the housing room and remained there for 28 days. Following this time period the animals were allocated into the midazolam treatment group (M-R1; $n=10)$, and the control group (S-R1; $n=10)$ which received a saline solution. The groups were matched for the open arm time measured in trial 1 to ensure equal variation in the treatment groups. The rats were injected $30 \mathrm{~min}$ before re-exposure to the EPM. This second trial was conducted in the same R1 as in trial 1 .

\section{EXPERIMENT 2: SWITCHED ROOM}

Another group of drug-naive rats $(n=20)$ was tested on the EPM in R1 for the first trial. As described in Experiment 1, the animals were allocated into the midazolam treatment group (M-R2; $n=10)$, and the control group (S-R2; $n=10)$, which received a saline solution. This time, however, the second trial was conducted in another room (R2), the switched room, to which the animals had previously not been exposed. All rats were injected $30 \mathrm{~min}$ before re-exposure.

\section{BEHAVIORAL ANALYSIS}

The standard measurements such as the time spent in the open arms and the number of entries in either the open or the closed arms were automatically analyzed by the software viewer ${ }^{2}$ (Biobserve $\mathrm{GmbH}$, Bonn, Germany). Entries were counted when all four paws had crossed into one of the arms. Horizontal locomotor activity is expressed as the number of closed arm entries and the actual distance $(\mathrm{cm})$ covered by the animal in the entire maze. Percentage of time spent on the open arms was calculated as percentage of the total time the rat spent on the maze [open arm time $\%=100 \times(\mathrm{OA}$ time/total time)]. Other behavioral variables such as the frequency of rearings (rat stood on its hind paws, raised both forepaws off the ground and ended when at least one forepaw had reached the floor again), head dips (dipping the head below the open arm of the EPM, with all four paws on an open arm), and risk assessment (at least one paw remains in a closed arm while nose and eyes cross into one of the open arm) were analyzed by an experienced experimenter blind to the treatment condition.

\section{STATISTICS}

The comparisons between the two trials in the pilot experiment, Experiment 1, and Experiment 2, respectively, were performed with repeated measures two-way ANOVAs and Tukey post hoc comparisons. Pearson's correlations were used to test for individual relationships between trials. Data are expressed as mean + SEM. All $P$-values are taken as statistically significant when $P \leq 0.05$.
The dose-response data was analyzed by two-tailed unpaired $t$-tests against the control group and the significance level was Bonferroni corrected to adjust for the increasing $\alpha$-error of multiple testing $(P<0.017)$.

\section{RESULTS \\ PILOT EXPERIMENT}

To assess the effect of an environmental change on EPM anxietylike behavior in re-test trial 2 following day 14 after trial 1, testing was carried out in either the same or an unfamiliar room. Under both conditions a significant decrease in open arm exploration was observed in the room change group (post hoc $P=0.01$ ) and without change in room (post hoc $P<0.001$ ) between trial 1 and 2 [room change: $F_{(1,34)}=1.76, P=0.19$; trial: $F_{(1,34)}=22.15, P<0.001$; interaction: $\left.F_{(1,34)}=0.30, P=0.59\right]$. A similar effect was not found for closed arm visits, [room change: $F_{(1,34)}=1.28, P=0.27$; trial: $F_{(1,34)}=2.42$, $P=0.13$; interaction: $F_{(1,34)}=0.44, P=0.51$; Figure 1].

The relationship between the open arm time measures in both trials was significant in the animals which were re-tested in the unfamiliar room $(r=0.48, P=0.04)$, and as a trend in the group tested twice in the same room $(r=0.44, P=0.07)$.

\section{DOSE-RESPONSE EXPERIMENT}

Due to the fact that a variety of concentrations of midazolam are cited in the available literature it was prudent to test for the effective dose in our set up (i.e., light condition) of the EPM. Of the three tested concentrations only the $0.25-\mathrm{mg} / \mathrm{kg}$ dose appeared to be anxiolytic. In fact, the higher doses $(0.5$ and $1.0 \mathrm{mg} / \mathrm{kg})$ had an increasingly sedative effect in that a high number of animals fell off the maze during the open arm exploration. Statistical testing revealed an anxiolytic effect only between saline controls and $0.25 \mathrm{mg} / \mathrm{kg}\left[t_{(13)}=-2.85 ; P=0.01 ;\right.$ Figure 2].

\section{EXPERIMENT 1: SAME ROOM}

The effect of the 28-days time span between trials conducted in the same room showed no significant changes in the percentage of time on the open arms [ANOVA: trial 1 vs. $2: F_{(1,18)}=2.84, P=0.11$;

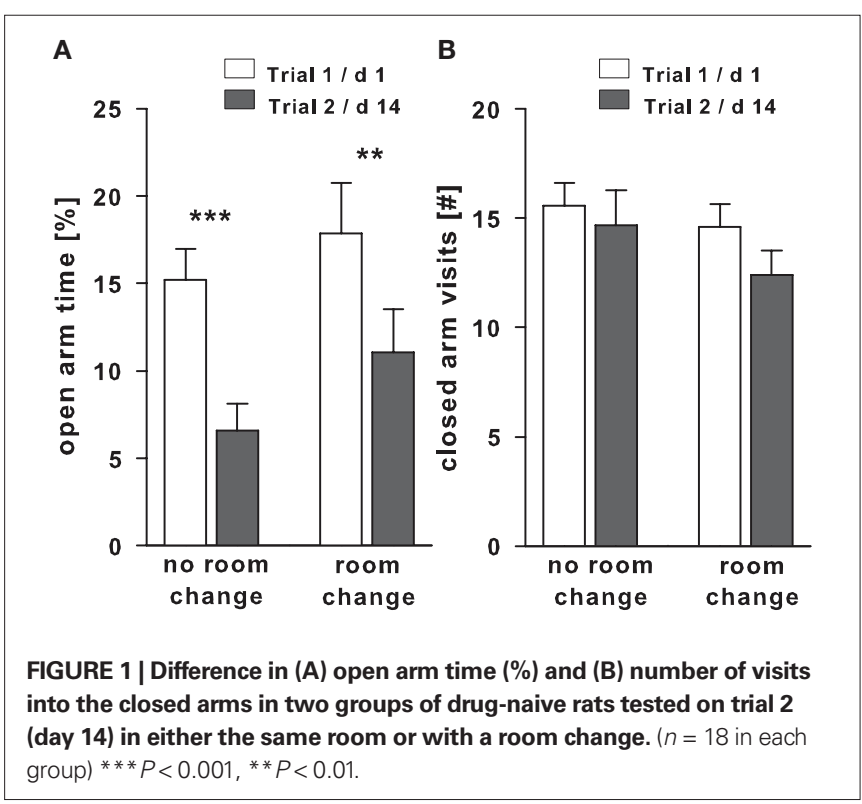


treatment saline vs. midazolam: $F_{(1,18)}=0.05, P=0.83$; interaction effect: $\left.F_{(1,18)}=0.36, P=0.56\right]$. The locomotor activity did not differ significantly between trials in both treatment groups after post hoc comparison [M-R1 $P=0.20$; S-R1 $P=0.11$; trial: $F_{(1,18)}=4.05$, $P=0.11$; treatment: $F_{(1,18)}=0.01, P=0.93$; interaction: $F_{(1,18)}=0.06$, $P=0.81]$. Similarly the closed arm visits remained statistically nonsignificant for both treatment groups [trial: $F_{(1,18)}=1.40, P=0.25$; treatment: $F_{(1,18)}=0.07, P=0.79$; interaction: $\left.F_{(1,18)}=1.13, P=0.30\right]$, while the rearing activity was significantly decreased only in the control animals [post hoc S-R1 $P=0.03$, trial 1: $11.70 \pm 0.99$; trial 2: $8.00 \pm 1.18 ; \mathrm{M}-\mathrm{R} 1 P=0.08$, trial 1: $11.70 \pm 1.39$, trial 2: $8.80 \pm 1.03$; trial: $F_{(1,18)}=8.850, P=0.008$; treatment: $F_{(1,18)}=0.11, P=0.74$; interaction: $\left.F_{(1,18)}=0.13, P=0.72\right]$. Additional parameters to assess

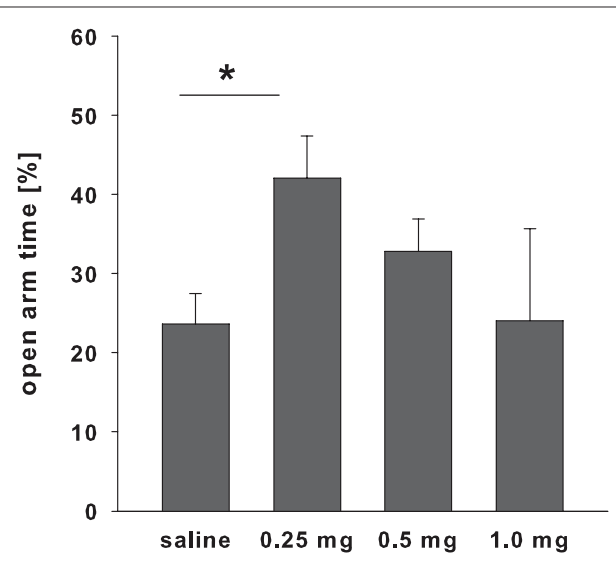

FIGURE 2 | Dose-response of three different doses of midazolam and saline controls on the open arm time (\%) of trial 1 on the EPM. Due to increased sedative action of the treatment the number of animals which completed a 5-min trial without falling off the maze decreased (saline $n=8$; $0.25 \mathrm{mg} n=7 ; 0.5 \mathrm{mg} n=5 ; 1.0 \mathrm{mg} n=3) .{ }^{*} P<0.017$. the emotional state in trial 2 did not differ from trial 1 in either the saline or midazolam treated animals for risk assessment [trial: $F_{(1,18)}=3.48, P=0.08$; treatment: $F_{(1,18)}=0.32, P=0.58$; interaction: $F_{(1,18)}=0.01, P=0.89$ ], and head dips [trial: $F_{(1,18)}=1.29$, $P=0.271$; treatment: $F_{(1,18)}=0.41, P=0.53$; interaction: $F_{(1,18)}=0.54$, $P=0.47$; Figure 3].

\section{EXPERIMENT 2: SWITCHED ROOM}

Compared to trial 1 , the additional change in room on trial 2 to the inter-trial time of 28 days showed a significant increase in percentage of time spent on the open arms in the midazolam treated animals $(\mathrm{M}-\mathrm{R} 2$ post hoc $P=0.05)$, while the saline treated group (S-R2 post hoc $P=0.31$ ) was unaffected [trial: $F_{(1,18)}=4.96, P=0.04$; treatment: $F_{(1,18)}=0.35, P=0.56$; interaction: $\left.F_{(1,18)}=0.55, P=0.47\right]$. The explorative activity, as measured in closed arm entries, was significantly elevated only in the midazolam treated group [post hoc: M-R2 $P=0.14$; S-R2 $P=0.02$; trial: $F_{(1,18)}=8.09, P=0.01$; treatment: $F_{(1,18)}=1.97, P=0.18$; interaction: $\left.F_{(1,18)}=0.47, P=0.50\right]$. While the distance covered revealed no significant changes between trials [trial: $F_{(1,18)}=1.65, P=0.22$; treatment: $F_{(1,18)}=0.03, P=0.86$; interaction: $\left.F_{(1,18)}=0.01, P=0.94\right]$. The rearing count was reduced significantly in both groups on trial 2 [post hoc M-R2 $P=0.01$, trial 1: $10.70 \pm 0.60$; trial 2: $6.80 \pm 0.71 ;$ S-R2 $P<0.001$, trial 1: $13 \pm 0.97$; trial 2: $7.40 \pm 0.96$; trial: $F_{(1,18)}=24.817, P<0.001$; treatment: $F_{(1,18)}=4.70, P=0.04$; interaction: $F_{(1,18)}=0.79, P=0.38$ ]. Risk assessment behavior remained unchanged between trials in both treatment groups [trial: $F_{(1,18)}=1.78, P=0.20$; treatment: $F_{(1,18)}=0.52, P=0.48$; interaction: $\left.F_{(1,18)}=2.61, P=0.12\right]$. However, the analysis of the number of head dips below the level of the open arms revealed a significantly increase in the $\mathrm{M}-\mathrm{R} 2$ group (post hoc $P=0.003$ ) on trial 2, while no difference was found in the S-R2 group [post hoc $P=0.79$; trial: $F_{(1,18)}=6.97, P=0.02$; treatment: $F_{(1,18)}=0.10$, $P=0.75$; interaction: $F_{(1,18)}=5.15, P=0.04$; Figure 4].
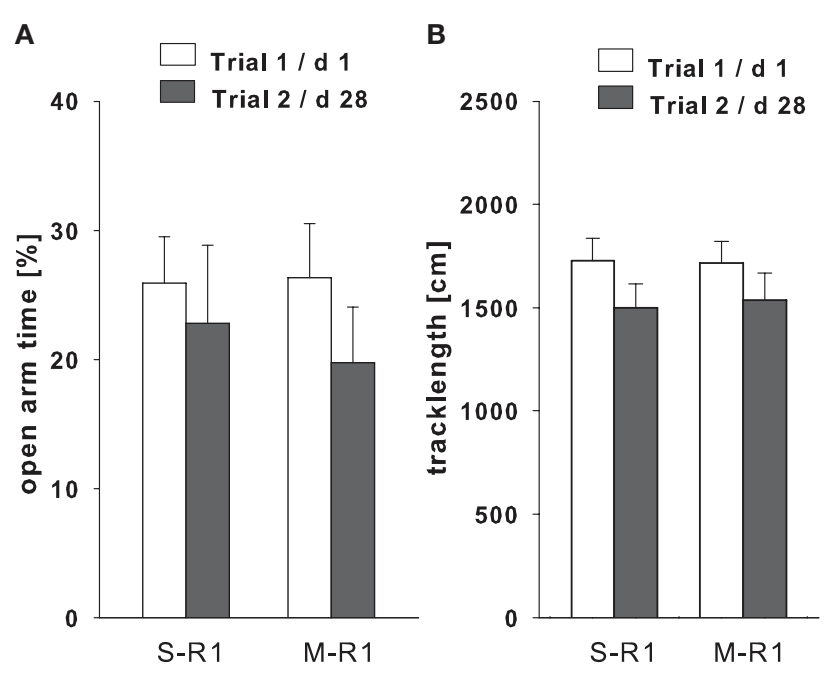

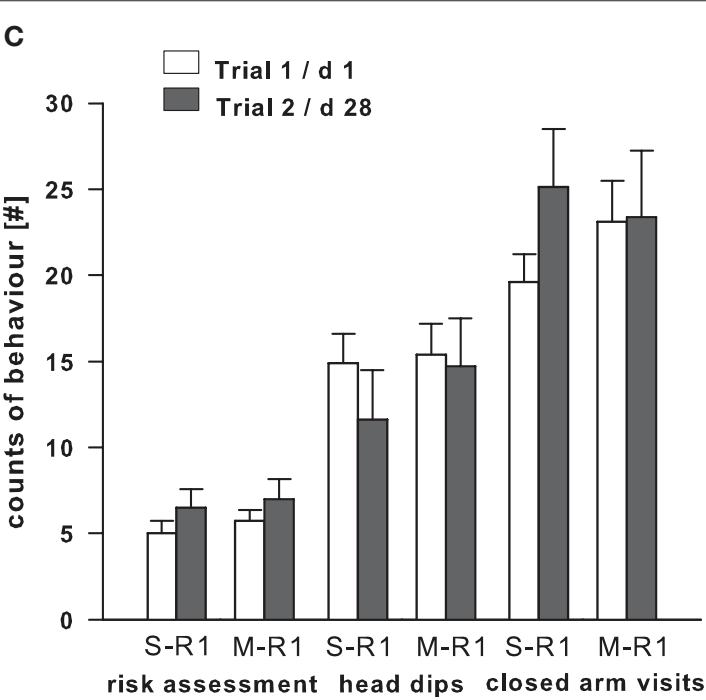

FIGURE 3 |Treatment effects in trial 2 (dark bars) in the EPM test compared to trial 1 (open bars) in the same room (R1) on (A) the percentage of time spent in the open arms, (B) explorative activity measured as track length on the entire maze, and (C) ethological behaviors, i.e., count of risk assessment, head dips, and rearings. Each animal was injected before the second trial with either saline (S-R1) or midazolam (M-R1; $0.25 \mathrm{mg} / \mathrm{kg}$ ), with $n=10$ in each group. ${ }^{*} P<0.05$. 

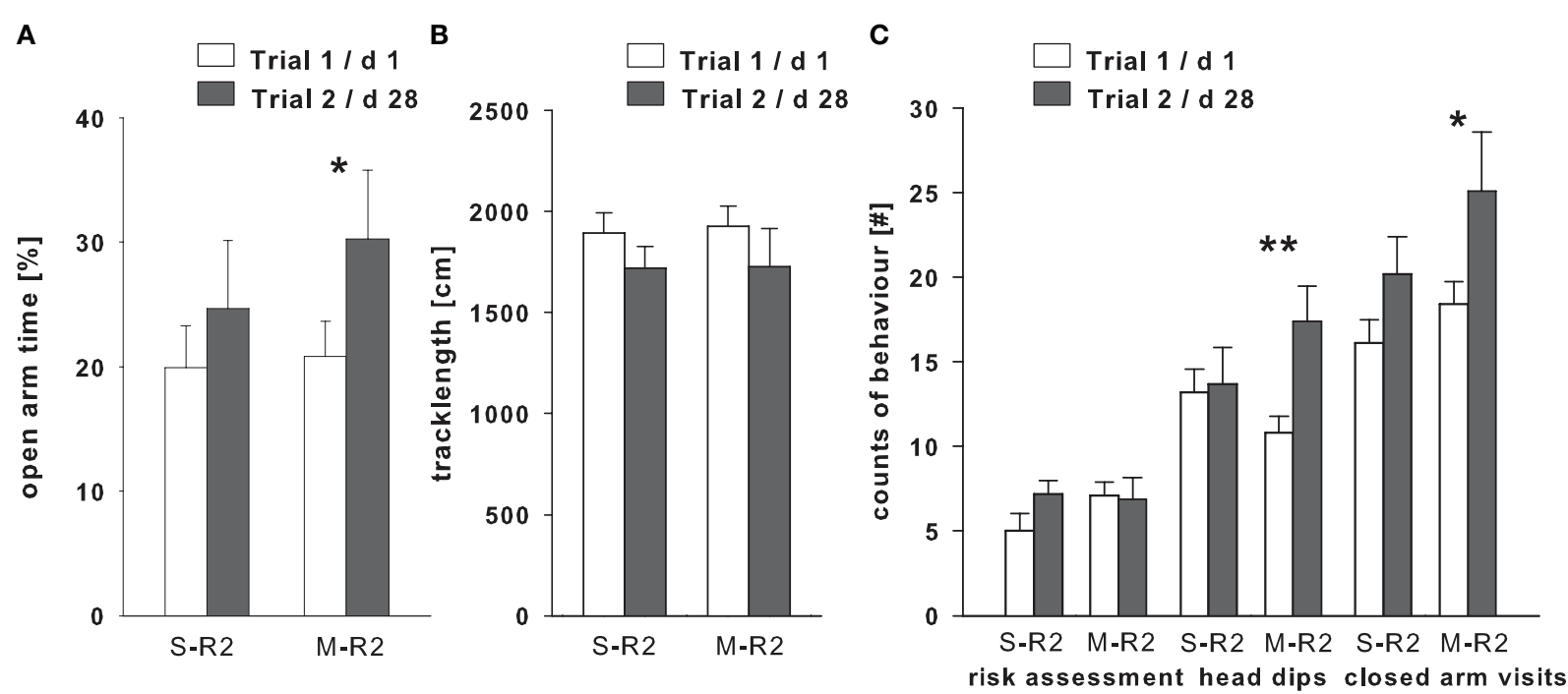

FIGURE 4 | Treatment effects in trial 2 (dark bars) in the EPM compared to a different testing room (R2) in trial 1 (light bars) on (A) the percentage of time spent in the open arms, (B) explorative activity measured as track length on the entire maze, and (C) ethological behaviors, i.e., count of risk assessment, head dips, and rearings. Each animal was injected before the second trial with either saline (S-R2) or midazolam (M-R2; $0.25 \mathrm{mg} / \mathrm{kg})$, with $n=10$ in each group. ${ }^{*} P<0.01,{ }^{*} P<0.05$.

\section{CORRELATIONS BETWEEN TRIALS}

To further analyze the relation between trial 1 and 2, correlation coefficients of conventional and the assessed ethological measurements of EPM behaviors are summarized in Table 1. The rationale for these analyses is the exploration of the reliability of individual behaviors between trials within each group and therefore to improve the construct validity of this re-test paradigm.

The percentage of time spent in and the number of visits into the open arms were highly correlated in the control animals in Experiment 1, but not in the midazolam treated group. Furthermore, only the saline treated group of rats showed significant correlation of ethological measures between trial 1 and 2. Formal measures of activity, i.e., visits into the closed arms and rearing behavior, did not show significant relationships except for the midazolam treated animals in Experiment 1. In Experiment 2, both treatment groups showed a significant positive correlation of the percentage of time spent in the open arms between trial 1 and trial 2. There were significant correlations found within the measure of risk assessing behavior, again in both treatment groups, when the room was changed in trial 2.

\section{DISCUSSION}

The results of both experiments show that an inter-trial interval of 28 days alone appears sufficient for a stable response upon re-testing in Wistar rats when treated with saline before trial 2 of the EPM. An extension of inter-trial intervals has previously been shown by us to re-instate the reactivity to the EPM to initial levels in terms of time spent on the open arms. In the study of Schwarting and Pawlak (2004) a period of 120 days between trials not only recovered the OA time but also showed a significant correlation between the two trials. Nevertheless, it is important to note that this period can apparently be reduced to 28 days as shown in the present study.
Table 1 | Correlations between trial 1 and trial 2 EPM measures: effects of midazolam $(0.25 \mathrm{mg} / \mathrm{kg})$ or saline on trial 2 for both experiments.

\begin{tabular}{lcccc}
\hline Trial 1/2 & \multicolumn{2}{c}{$\begin{array}{c}\text { Experiment 1 } \\
\text { (same) }\end{array}$} & \multicolumn{2}{c}{$\begin{array}{c}\text { Experiment 2 } \\
\text { (switch) }\end{array}$} \\
\cline { 2 - 5 } & S-R1 & M-R1 & S-R2 & M-R2 \\
\hline OA time \% & $0.857^{* *}$ & 0.428 & $0.596^{*}$ & $0.559^{*}$ \\
OA visits & $0.910^{* *}$ & 0.196 & 0.217 & 0.457 \\
CA visits & -0.084 & $0.625^{*}$ & 0.263 & 0.476 \\
Rearings & 0.085 & 0.088 & -0.517 & 0.037 \\
Risk assessment & -0.491 & 0.472 & $0.559^{*}$ & $0.551^{*}$ \\
Head dips & $0.719^{* *}$ & 0.274 & 0.405 & 0.516 \\
Grooming & 0.186 & 0.000 & -0.371 & 0.229 \\
\hline
\end{tabular}

Pearson correlation coefficient (one-tailed) ${ }^{*} P<0.01, * P<0.05$.

Most importantly, the increase of the percentage of time spent on the open arms found only in the animals treated with midazolam $(0.25 \mathrm{mg} / \mathrm{kg})$ in Experiment 2 suggests that benzodiazepines do not lose their anxiolytic-like effects upon re-testing in the EPM. Furthermore, the saline treated groups of either testing condition showed no significant decrease in open arm time. Moreover, only in the switched room condition did the midazolam group show a positive correlation between both trials, indicating that the relative open arm times were comparable between trials and also on an individual level. Our testing paradigm to introduce an environmental change outside of the EPM apparatus instead of using proximal cues (Pereira et al., 1999) appears suitable as a quick and easy way for the prevention of the OTT. The increased open arm exploration, i.e., time spent on the open arms and head dips over the edges, is part of the usual effect of benzodiazepines, when given before trial 1 . 
It has been frequently reported that benzodiazepines lose their efficacy when injected before trial 2 (File and Zangrossi Jr., 1993; Rodgers and Shepherd, 1993; Cruz-Morales et al., 2002). An emotional shift in the animal has been suggested as a probable explanation; the rodent switches from initial exploration of the maze to learned avoidance of the open arms even during the last minutes of the first trial, and shifts into an acquired phobiclike state during a later, second exposure to the EPM (Lister, 1987; File, 1993; File and Zangrossi Jr., 1993; Cruz-Morales et al., 2002). Furthermore, the initial motivational approach/avoidance conflict situation is attenuated in a second exposure to the maze because the arms have been explored and the avoidance of the exposed areas has been learned. Introducing a motivational conflict in the second trial, i.e., hot air stream in one closed arm, was able to maintain the exploration on the initial level and abolish the OTT (Pereira et al., 1999). Our results show that an intertrial delay of 28 day combined with a change of room was able to retain the anxiolytic-like effects of midazolam on the percentage of time spent on the open arms in the M-R2 switched room group. In addition, this group showed a substantial correlation between both trials for the open arm time percentage underlining the relation between both trials and indicating stable trait behavior. In parallel, we found a stable and correlated behavior of both trials in the control animals (S-R2, switched room) with no significant decrease of exploration (percentage of open arm time, closed arm visits). Previous findings reported that the OTT is evident with an inter-trial time of $24 \mathrm{~h}$ up to 14 days (File et al., 1990), but has never been tested with longer delays to the best of our knowledge.

Furthermore, even though there have been reports that the use of a different room 3 weeks after the initial exposures allows for repeated measures of plus-maze behavior, these reports unfortunately lack sufficient data on an individual level as well as a pharmacological validation (Adamec and Shallow, 2000; Adamec and Young, 2000). On the other hand the results of a study investigating a 24-h re-test of the EPM under differing conditions found the change of laboratory room insufficient to alter the learned avoidance of the open arms. It was suggested that the proximal cues of the maze itself would overshadow the distal cues of an unfamiliar room (Rodgers et al., 1997). The two rooms used in this study differed mainly in size, but had no additional cues on the walls, and were located in the same direction and distance from the keeping rooms of the rats. Taken together, these facts make it conceivable that combining the change of the testing environment (i.e., rooms) and the period of time between trials (here, 28 days) is sufficient to maintain the approach/avoidance conflict, and both appear crucial for the abolishment of the OTT. This is further supported by the results of Experiment 1 when the EPM remained in the original room (R1) for the second trial (same condition). Here, the time period between trials alone was not able to preserve the anxiolytic-like effects of midazolam and therefore did not modify the phenomenon of OTT. Surprisingly, the behavior of the control group (S-R1) in trial 2 did not differ significantly from the initial measurements taken on trial 1. The high number of complex ethological parameters which correlated over 28 days inter-trial time could indicate the measurement of actual trait-anxiety on the EPM, and was to a lesser extent also seen in the control animals of the R2-group (switched room). In both experiments, the behavior of the control groups appears to be stable over time and on an individual level supporting previous studies reporting trait-anxiety-like behavior in a re-test paradigm (Schwarting and Pawlak, 2004; Pawlak et al., 2008). It is important to note that even though the individuals showed a significant correlation of time spent on the open arms in test/re-test situations in HOA and LOA Wistar rats, a marked decrease of open arm exploration was occurring within $24 \mathrm{~h}$. This was recovered only then a second animal group was exposed to the EPM a 120-days after the first trial (Schwarting and Pawlak, 2004).

In the pilot experiment using only untreated rats, the combination of an inter-trial delay of 14 days (a period in which the OTT is still in effect, File, 1993) and a change in testing rooms was not sufficient to maintain the baseline level of exploration on the open arms shown in trial 1. Even though Figure 1A shows a slight increase of open arm time in the switched room group compared to the same room group, this was not found to be statistically significant. However, the change in room appeared to amplify the correlative relationship between the trials, indicating a recovery of a stable level of open arm exploration on an individual level as was seen in the subsequent experiments when trial 2 was carried out 28 days after trial 1. Additionally, the fact that with exception of the M-R2 group the general locomotor activity remained unchanged across the testing conditions with 14 days as well as 28 days between trials, suggests that changes in open arm time exploration are not due to a general activation of locomotion. Moreover, both rooms used in this study are regularly employed when using the EPM and yield comparable results.

The results of the present study could imply a change in memory retrieval on trial 2 and therefore the perception of the EPM over a time course longer than 14 days. Even though it could be argued that the decreased rearing activity across all treatment groups and test situations implies the recognition of the maze itself, as rearing has been suggested to show reactivity to new stimuli (Pawlak and Schwarting, 2002).

Additionally, the measurements of other and more complex ethological relevant parameters (risk assessment and head dips) show similar treatment effects of midazolam on trial 2 after 28 days of delay to an initial treatment on the first trial (AlbrechetSouza and Brandao, 2010). The inclusion of these so called "novel" ethological measurements allows for a broader assessment of the behavioral repertoire shown in trial $1 /$ trial 2 protocol. In view of re-instating the initial situation, the additional emotional dimensions should be considered. It has been shown that these measurements load differently on independent factors in re-test paradigms with 24-48 h inter-trial delays (File and Zangrossi Jr., 1993; Albrechet-Souza et al., 2008) indicating that the behavioral profile of the rodent changes: Shifting from unconditioned anxiety to learned avoidance/fear. Our results show significant correlations of risk assessing behavior in both treatment groups in the switched room testing condition (M-R2 and S-R2), but not when the EPM was re-tested in the same room. This implicates strongly that the motivational conflict between approaching the unknown areas and avoiding potentially dangerous open arms is re-instated in trial 2 and is dependent on the changed environment of Experiment 2. 
In summary, we suggest that a time period of 28 days in combination with a change in testing rooms is necessary to reliably re-test EPM behavior and to "re-set" the animal's behavior to a state comparable to trial 1 . Therefore, we propose this modified EPM procedure for re-testing EPM behavior to be applied for any longitudinal study design, especially for testing new pharmacological compounds, or after conditional genetic manipulations.

\section{REFERENCES}

Adamec, R., and Shallow, T. (2000). Effects of baseline anxiety on response to kindling of the right medial amygdala. Physiol. Behav. 70, 67-80.

Adamec, R., and Young, B. (2000). Neuroplasticity in specific limbic system circuits may mediate specific kindling induced changes in animal affect-implications for understanding anxiety associated with epilepsy. Neurosci. Biobehav. Rev. 24, 705-723.

Albrechet-Souza, L., Borelli, K. G., and Brandao, M. L. (2008). Activity of the medial prefrontal cortex and amygdala underlies one-trial tolerance of rats in the elevated plus-maze. J. Neurosci. Methods 169, 109-118.

Albrechet-Souza, L., and Brandao, M. L. (2010). Hormonal and cognitive factors associated with the exploratory behavior of rats submitted to repeated sessions of the elevated plus-maze. Psychol. Neurosci. 3, 43-52.

Andreatini, R., and Bacellar, L. F. (2000). Animal models: trait or state measure? The test-retest reliability of the elevated plus-maze and behavioral despair. Prog. Neuropsychopharmacol. Biol. Psychiatry 24, 549-560.

Calzavara, M. B., Patti, C. L., Lopez, G. B., Abilio, V. C., Silva, R. H., and FrussaFilho, R. (2005). Role of learning of open arm avoidance in the phenomenon of one-trial tolerance to the anxiolytic effect of chlordiazepoxide in mice. Life Sci. 76, 2235-2246.

Carobrez, A. P., and Bertoglio, L. J. (2005). Ethological and temporal analyses of anxiety-like behavior: the elevated plus-maze model 20 years on. Neurosci. Biobehav. Rev. 29, 1193-1205.

Chesler, E. J., Wilson, S. G., Lariviere, W. R., Rodriguez-Zas, S. L., and Mogil, J.S. (2002). Influences of laboratory environment on behavior. Nat. Neurosci. 5, 1101-1102.

Cruz-Morales, S. E., Santos, N. R., and Brandao, M. L. (2002). One-trial tolerance to midazolam is due to enhancement of fear and reduction of anxiolytic-sensitive behaviors in the elevated plus-maze retest in the rat. Pharmacol. Biochem. Behav. 72, 973-978.

Dal-Col, M. L., Pereira, L. O., Rosa, V. P., Calixto, A. V., Carobrez, A. P., and Faria, M. S. (2003). Lack of midazolam-induced anxiolysis in the plus-maze trial 2 is dependent on the length of trial 1. Pharmacol. Biochem. Behav. 74, 395-400.

File, S.E. (1993). The interplay of learning and anxiety in the elevated plus-maze. Behav. Brain Res. 58, 199-202.

File, S. E., Mabbutt, P. S., and Hitchcott, P. K. (1990). Characterisation of the phenomenon of "one-trial tolerance" to the anxiolytic effect of chlordiazepoxide in the elevated plus-maze. Psychopharmacology (Berl.) 102, 98-101.

File, S. E., and Zangrossi, H. Jr. (1993). "One-trial tolerance" to the anxiolytic actions of benzodiazepines in the elevated plus-maze, or the development of a phobic state? Psychopharmacology (Berl.) 110, 240-244

File, S. E., Zangrossi, H. Jr., Viana, M., and Graeff, F. G. (1993). Trial 2 in the elevated plus-maze: a different form of fear? Psychopharmacology (Berl.) 111, 491-494.

Frussa-Filho, R., Barbosa-Junior, H., Silva, R. H., Da, C. C., and Mello, C. F. (1999). Naltrexone potentiates the anxiolytic effects of chlordiazepoxide in rats exposed to novel environments. Psychopharmacology (Berl.) 147, 168-173.

Gonzalez, L. E., and File, S. E. (1997). A five minute experience in the elevated plus-maze alters the state of the benzodiazepine receptor in the dorsal raphe nucleus. J. Neurosci. 17, 1505-1511.

Hascoet, M., Bourin, M., and Couetoux du, T. A. (1997). Influence of prior experience on mice behavior using the four-plate test. Pharmacol. Biochem. Behav. 58, 1131-1138.

Holmes, A., Iles, J. P., Mayell, S. J., and Rodgers, R. J. (2001). Prior test experience compromises the anxiolytic efficacy of chlordiazepoxide in the mouse light/dark exploration test. Behav. Brain Res. 122, 159-167.

Holmes, A., and Rodgers, R. J. (1998). Responses of Swiss-Webster mice to repeated plus-maze experience: further evidence for a qualitative shift in emotional state? Pharmacol. Biochem. Behav. 60, 473-488.

Holmes, A., and Rodgers, R. J. (1999). Influence of spatial and temporal

\section{ACKNOWLEDGMENTS}

The authors would like to thank Hanna Thiery for her excellent work during experiments and animal handling. This work was supported by grants from the German Research Foundation (DFG PA 818/4-1), National Science Council of the ROC (NSC 98-2410H-040-003; NSC 99-2410-H-241-004; NSC 99-2410-H-040-008), and the Bundesministerium für Bildung und Forschung (BMBF FKZ: 01GS08152; Spanagel et al., 2010).

manipulations on the anxiolytic efficacy of chlordiazepoxide in mice previously exposed to the elevated plus-maze. Neurosci. Biobehav. Rev. 23, 971-980.

Lamprea, M. R., Cardenas, F. P., Silveira, R., Morato, S., and Walsh, T. J. (2000). Dissociation of memory and anxiety in a repeated elevated plus maze paradigm: forebrain cholinergic mechanisms. Behav. Brain Res. 117, 97-105.

Landgraf, R., and Wigger, A. (2002). High vs low anxiety-related behavior rats: an animal model of extremes in trait anxiety. Behav. Genet. 32, 301-314.

Lister, R. G. (1987). The use of a plusmaze to measure anxiety in the mouse. Psychopharmacology (Berl.) 92, 180-185.

Pawlak, C. R., Ho, Y. J., and Schwarting, R. K. (2008). Animal models of human psychopathology based on individual differences in novelty-seeking and anxiety. Neurosci. Biobehav. Rev. 32, 1544-1568.

Pawlak, C. R., Karrenbauer, B. D., Schneider, P., and Ho, Y. J. (in press). The elevated plus-maze test: differential psychopharmacology of anxietyrelated behaviour. Emot. Rev.

Pawlak, C. R., and Schwarting, R. K. (2002). Object preference and nicotine consumption in rats with high vs. low rearing activity in a novel open field. Pharmacol. Biochem. Behav. 73, 679-687.

Pellow, S., Chopin, P., File, S. E., and Briley, M. (1985). Validation of open:closed arm entries in an elevated plus-maze as a measure of anxiety in the rat. J. Neurosci. Methods 14, 149-167.

Pereira, J. K., Vieira, R. J., Konishi, C. T., Ribeiro, R. A., and Frussa-Filho, R. (1999). The phenomenon of "one-trial tolerance" to the anxiolytic effect of chlordiazepoxide in the elevated plus-maze is abolished by the introduction of a motivational conflict situation. Life Sci. 65, L101-L107.

Rodgers, R. J., Johnson, N. J., Carr, J., and Hodgson, T. P. (1997). Resistance of experientially-induced changes in murine plus-maze behaviour to altered retest conditions. Behav. Brain Res. 86, 71-77.
Rodgers, R. J., Johnson, N. J., Cole, J. C., Dewar, C.V., Kidd, G. R., and Kimpson, P. H. (1996). Plus-maze retest profile in mice: importance of initial stages of trail 1 and response to post-trail cholinergic receptor blockade. Pharmacol. Biochem. Behav. 54, 41-50.

Rodgers, R. J., Lee, C., and Shepherd, J. K. (1992). Effects of diazepam on behavioural and antinociceptive responses to the elevated plus-maze in male mice depend upon treatment regimen and prior maze experience. Psychopharmacology (Berl.) 106, 102-110.

Rodgers, R. J., and Shepherd, J. K. (1993). Influence of prior maze experience on behaviour and response to diazepam in the elevated plus-maze and light/dark tests of anxiety in mice. Psychopharmacology (Berl.) 113, 237-242.

Rosa, V. P., Vandresen, N., Calixto, A. V., Kovaleski, D. F., and Faria, M. S. (2000). Temporal analysis of the rat's behavior in the plus-maze: effect of midazolam. Pharmacol. Biochem. Behav. 67, 177-182.

Schwarting, R. K., and Pawlak, C. R. (2004). Behavioral neuroscience in the rat: taking the individual into account. Methods Find. Exp. Clin. Pharmacol. 26, 17-22.

Spanagel, R., Bartsch, D., Brors, B., Dahmen, N., Deussing, J., Eils, R., Ende, G., Gallinat, J., GebickeHaerter, P., Heinz, A., Kiefer, F., Jäger, W., Mann, K., Matthäus, F., Nöthen, M., Rietschel, M., Sartorius, A., Schütz, G., Sommer, W. H., Sprengel, R., Walter, H., Wichmann, E., Wienker, T., Wurst, W., and Zimmer, A. (2010). An integrated genome research network for studying the genetics of alcohol addiction. Addict. Biol. 15, 369-379.

Wahlsten, D., Metten, P., Phillips, T. J., Boehm, S. L., Burkhart-Kasch, S., Dorow, J., Doerksen, S., Downing, C., Fogarty, J., Rodd-Henricks, K., Hen, R., McKinnon, C.S., Merrill, C. M., Nolte, C., Schalomon, M., Schlumbohm, J. P., Sibert, J. R., Wenger, C. D., Dudek, B. C., and Crabbe, J. C. (2003). Different data from different labs: lessons from studies of gene-environment interaction. J. Neurobiol. 54, 283-311. 
Walf, A. A., and Frye, C.A. (2007). The use of the elevated plus maze as an assay of anxiety-related behavior in rodents. Nat. Protoc. 2, 322-328.

Conflict of Interest Statement: The authors declare that the research was conducted in the absence of any commercial or financial relationships that could be construed as a potential conflict of interest.

Received: 08 March 2011; accepted:08 July 2011; published online: 27 July 2011.
Citation: Schneider P, Ho Y-J, Spanagel R and Pawlak CR (2011) A novel elevated plus-maze procedure to avoid the one-trial tolerance problem. Front. Behav. Neurosci. 5:43. doi: 10.3389/fnbeh.2011.00043

Copyright (c) 2011 Schneider, Ho, Spanagel, Pawlak. This is an open-access article subject to a non-exclusive license between the authors and Frontiers Media $S A$, which permits use, distribution and reproduction in other forums, provided the original authors and source are credited and other Frontiers conditions are complied with. 